\title{
Treatment Patterns and Economic Assessment of Systemic Therapy for Metastatic Colorectal Cancer
}

\author{
Thomas H. Cartwright1, Lonnie K. Wen², Robyn K. Harrell' ${ }^{1}$, Patricia S. Fox ${ }^{1}$, Janet L. Espirito ${ }^{{ }^{*}}$, \\ Ed Wang ${ }^{2}$
}

${ }^{1}$ The US Oncology Network, McKesson Specialty Health, Houston, TX, USA

${ }^{2}$ Bayer Healthcare, Whippany, NJ, USA

Email: *thomas.cartwright@usoncology.com

\begin{abstract}
How to cite this paper: Cartwright, T.H., Wen, L.K., Harrell, R.K., Fox, P.S., Espirito, J.L. and Wang, E. (2016) Treatment Patterns and Economic Assessment of Systemic Therapy for Metastatic Colorectal Cancer. Journal of Cancer Therapy, 7, 646-655. http://dx.doi.org/10.4236/jct.2016.79067
\end{abstract}

Received: August 13, 2016

Accepted: September 9, 2016

Published: September 12, 2016

Copyright $\odot 2016$ by authors and Scientific Research Publishing Inc. This work is licensed under the Creative Commons Attribution International License (CC BY 4.0).

http://creativecommons.org/licenses/by/4.0/ (c) (i) Open Access

\begin{abstract}
Introduction: Colorectal cancer (CRC) is the third most commonly diagnosed cancer and the third leading cause of cancer deaths in the United States. The goal of this study was to understand treatment patterns, biomarker testing practices, treatment adherence, and the clinical and economic outcomes associated with chemotherapy for metastatic disease. Methods and Materials: We retrospectively examined electronic health records of patients with metastatic CRC who initiated chemotherapy between 01 January 2007 and 30 June 2011, with follow-up to 30 June 2012. Parameters analyzed included demographics and clinical characteristics, treatment patterns, clinical outcomes, and health care resource utilization. Results: In the analysis, 756 patients were included; median age was 61 years (55\% male) at start of first line therapy. The most commonly used regimens in the first, second, and third line were FOLFOX + bevacizumab (46\%), FOLFIRI + bevacizumab (23\%), and irinotecan + cetuximab (23\%) respectively. Adherence to guidelines decreased with increasing line of therapy. When assessed by treatment backbone categories in the third line, outcome measures including overall survival (OS), and time to treatment discontinuation (TTD) were not statistically different between groups. In the multivariable model, body mass index (BMI), performance status, and KRAS were significant predictors of survival. Conclusions: This study provides insight into patterns of care and outcomes of $\mathrm{mCRC}$ patients for the aforementioned time period. As treatment options for mCRC evolve, it is valuable to understand the continuum of care to help inform future treatment among candidates for continued therapy.
\end{abstract}

\section{Keywords}

Metastatic Colorectal Cancer, Economic Assessment, Systemic Therapy, Treatment Patterns 


\section{Introduction}

Colorectal cancer (CRC) is the third most commonly diagnosed cancer and third leading cause of cancer-related deaths in the US. It is estimated that there will be more than 132,000 new cases and almost 50,000 deaths in 2015 [1]. The median age at diagnosis is 68 years [2]. The majority of patients are diagnosed with regional Stage II to III disease; however more than $50 \%$ will develop distant metastases. The estimated 5-year survival for distant metastatic disease is $13.1 \%$ [2].

The number of treatment options for metastatic CRC (mCRC) has increased in recent years, providing new combination chemotherapy and targeted therapy options [3] and improved overall survival. Yet survival remains poor for patients with advanced stage cancer. National organizations such as the National Comprehensive Cancer Network (NCCN) and cancer treatment organizations such as The US Oncology Network (USON) have developed guidelines to support the use of evidence-based treatment. The US Oncology Pathways are a subset of the NCCN guidelines. Despite the presence of guidelines, treatment selection in $\mathrm{mCRC}$ is dependent on many factors, including the treatment goals (e.g. surgical resection), toxicity, prior therapy type, molecular phenotype, patient performance status, age, comorbidities, and insurance coverage. Moderate to severe comorbidity, being uninsured, having rectal cancer, older age, and advanced tumor stage are reported as being associated with an increased risk of receiving treatment that is non-adherent to NCCN guidelines. Hines et al. reported that treatment non-adherence was associated with 3.6 times the risk of death in patients with CRC.

Although guideline recommendations for the treatment of mCRC exist, they often depend on prior treatments received, and lines of therapy are often blurred. Genetic alterations in tumor biomarkers such as KRAS and NRAS are also drivers of treatment selection in mCRC [3], and the NCCN guidelines strongly recommend genotyping all patients for RAS (KRAS, NRAS) and BRAF at diagnosis of stage IV disease. Approximately $40 \%$ of colorectal cancers have mutations in the KRAS gene, which is predictive of lack of response to the therapies targeted to the epidermal growth factor receptor (EGFR) [4]. Only tumors with wild-type KRAS are recommended to receive treatment with panitumumab and cetuximab. Because patients with mutant KRAS are not candidates for these therapies, fewer options exist for this population. Improved survival has been demonstrated with the use of EGFR inhibitors in patients with wild-type mCRC, and it has also been shown that survival is worse with use of these treatments in patients with KRAS mutations. Therefore use of these agents in patients without a known target can result in worse outcomes, exposure to unnecessary toxicity, and added cost.

Many studies of mCRC therapy have been performed in the first- and second-line treatment setting. A study by Hoverman et al. [5] looking at pathways, outcomes, and costs in mCRC reported lower overall costs with the use of pathways with survival comparable to the published literature. However, less information is available on outcomes with later lines of therapy. Median overall survival for studies in the chemorefractory, third-line and greater treatment settings have ranged from 5 to 10 months, depending on treatment [6]. 
As the treatment landscape and options for mCRC continue to evolve, it is important to understand how treatments are used in the continuum of care, to maximize benefit among patients who are candidates for continued therapy in advanced disease. Many factors influence subsequent treatment selection strategies in later lines of therapy for advanced CRC. Choice of initial therapy influences subsequent treatment, as does biomarker status. Given the blurred lines in treatment and the number of sequencing options available in later lines of therapy, the objectives of this study were to describe treatment patterns of patients with mCRC treated with multiple lines of therapy, to understand adherence to the NCCN guidelines and the USON Pathways, and to determine KRAS biomarker testing patterns. As new treatments become approved for later lines of therapy, and little information exists on utilization and outcomes in advanced disease, we focused on outcomes in the third-line of therapy, including clinical outcomes associated with different treatment categories, and costs of outpatient health care resource use associated with treatment.

\section{Patients and Methods}

This was a retrospective observational cohort study utilizing The USON iKnowMed ${ }^{\mathrm{TM}}$ electronic health record (EHR) database. Data were collected via programmatic queries of the database. The USON of community oncology practices is affiliated with approximately 1000 physicians across 19 states. Vital status data was supplemented with information from the US Department of Social Security death index.

The study population included adult patients with a diagnosis of mCRC initiating first-line chemotherapy from 01 January 2007 to 30 June 2011 and initiating a third-line therapy before 30 June 2012. Patient follow-up evaluation continued through 30 June 2012, for a minimum potential follow-up of 12 months. Patients may have been newly diagnosed metastatic or those diagnosed with earlier stage disease that became metastatic. Patients were excluded if they were: $<18$ years of age, enrolled in a clinical trial during the study period, or had another cancer diagnosis during the study period. Systemic treatments and lines of therapy from the programmatic queries were organized into a timeline for each patient, and regimens were reviewed and categorized into consistent categories for treatment and lines of therapy.

The NCCN Guidelines for Colon Cancer (version 3.2013) were used to determine adherence to clinical guidelines during this study. Adherence to The USON Pathways was assessed using version 2013-v2. Clinical trial regimens and best supportive care were considered "on pathway", but were not included in this assessment. Regimens were assessed retrospectively at the time of study based on the 2013 version of both NCCN and USON guidelines, as major changes in chemotherapy treatment recommendations were not observed to occur during that time.

Patients, including both commercially insured and Medicare patients, were matched from the EHR to the USON claims database for assessment of health care resource utilization and costs. Costs were calculated using submitted claims between the initiation of third-line therapy to the earliest endpoint of last contact, death, or progression up to 
the date of last chemotherapy plus 30 days, to estimate the cost of care during active treatment of third-line therapy. As all data was programmatically extracted from the EHR, progression was defined if a patient was identified as having a subsequent line of therapy. As submitted claims were for outpatient services, costs of hospitalizations and inpatient services were not included. All costs were calculated using 2012 Center for Medicare and Medicaid Services (CMS) physician fee schedules and average sales price (ASP) files reflecting a societal and payer perspective.

The Kaplan-Meier method was used to estimate overall survival and time to treatment discontinuation from initiation of third-line therapy. Log-rank tests were used to test for differences in outcomes by groups. P-values less than 0.05 were considered statistically significant and not adjusted for multiple comparisons. A generalized linear regression model was used to model cost in univariate and multivariable analysis as a function of third-line covariates, using the log link and gamma family. Chi square tests were used to compare categorical variables. The Wilcoxon rank-sum test was used to compare observed cost by adherence status. Statistical analyses were performing using SAS 9.4 (SAS Institute, Cary, NC, USA).

\section{Results}

\subsection{Demographic Characteristics and Treatment Patterns}

The study included 756 patients with a diagnosis of mCRC who received a first line of therapy and met the EHR eligibility criteria during the study period. Table 1 presents a summary of patient characteristics. The median age of the population at the start of first-line therapy was 61 years (range: 26 - 93 years). Fifty-five percent of the study population was male, and $39 \%$ of the population had Medicare as their primary payer. At the start of first-line therapy, $97 \%$ of patients had a ECOG performance status (PS) available; of these patients, $21 \%$ were PS $0,65 \%$ were PS 1 , and $11 \%$ were PS $2+$. There were 743 patients who received second-line therapy, and 577 patients received thirdline therapy.

In this patient population, the regimen (and backbone treatment categories) most frequently used for first-line therapy was FOLFOX + bevacizumab (oxaliplatin-based), for second-line therapy was FOLFIRI + bevacizumab (irinotecan-based) and for thirdline irinotecan + cetuximab (irinotecan + EGFR inhibitor). Table 2 presents a summary of the third-line backbone treatment categories. Median time to treatment discontinuation was 4.6, 3.0, and 2.3 months respectively for first-, second-, and third-line therapy periods.

Adherence to NCCN guidelines and USON Pathways was $88 \%$ and $84 \%$ respectively for first-line, $75 \%$ and $62 \%$ for second-line, and $55 \%$ and $38 \%$ for third-line therapy. Specifically in the third-line setting, for those who received irinotecan + cetuximab, the median number of cycles was $3 \%$ and $6 \%$ of these patients started at lower than standard doses.

There were 493 mCRC patients (65\%) with documented KRAS status during the study, with most patients having a documented status prior to third-line treatment. Of 
Table 1. Patient demographic and clinical characteristics.

\begin{tabular}{|c|c|}
\hline Patient characteristics & $\mathrm{N}=756$ \\
\hline \multicolumn{2}{|l|}{ Age at start of first-line therapy (years) } \\
\hline Mean (SD) & $60.8(12.6)$ \\
\hline Median & 61.2 \\
\hline Range (minimum - maximum) & $26.2-93.3$ \\
\hline \multicolumn{2}{|l|}{ Age at start of first-line therapy, $n(\%)$} \\
\hline$\geq 18-<50$ years & $156(20.6)$ \\
\hline$\geq 50-<65$ years & $304(40.2)$ \\
\hline$\geq 65$ years & $296(39.2)$ \\
\hline \multicolumn{2}{|l|}{ Gender, n (\%) } \\
\hline Male & $418(55.3)$ \\
\hline Female & $338(44.7)$ \\
\hline \multicolumn{2}{|l|}{ Diagnosis, n (\%) } \\
\hline Colon & $583(77.1)$ \\
\hline Rectal & $172(22.8)$ \\
\hline Both & $1(0.1)$ \\
\hline \multicolumn{2}{|l|}{ Body Mass Index, n (\%) } \\
\hline Normal & $328(43.4)$ \\
\hline Overweight & $255(33.7)$ \\
\hline Obese & $173(22.9)$ \\
\hline \multicolumn{2}{|l|}{ ECOG at index date $1, \mathrm{n}(\%)$ of $756 \mathrm{LL}$ patients } \\
\hline 0 & $161(21.3)$ \\
\hline 1 & $488(64.6)$ \\
\hline$\geq 2$ & $85(11.2)$ \\
\hline Unknown & $22(2.9)$ \\
\hline \multicolumn{2}{|l|}{ ECOG at index date $2, \mathrm{n}(\%)$ of $7432 \mathrm{~L}$ patients } \\
\hline 0 & $112(15.1)$ \\
\hline 1 & $510(68.6)$ \\
\hline$\geq 2$ & $99(13.3)$ \\
\hline Unknown & $22(2.9)$ \\
\hline \multicolumn{2}{|l|}{ ECOG at index date $3, \mathrm{n}(\%)$ of $5773 \mathrm{~L}$ patients } \\
\hline 0 & $58(10.1)$ \\
\hline 1 & $396(68.6)$ \\
\hline$\geq 2$ & $106(18.4)$ \\
\hline Unknown & $17(3.0)$ \\
\hline \multicolumn{2}{|l|}{ Metastasis location at start of first-line therapy, $\mathrm{n}(\%)$} \\
\hline Liver only & $214(28.3)$ \\
\hline Lung only & $24(3.2)$ \\
\hline Bone, ovary, peritoneum, or retroperitoneum only & $20(2.6)$ \\
\hline
\end{tabular}




\section{Continued}

Multiple sites

Others

Unknown

US geographic region, $\mathrm{n}(\%)$

South

West

Midwest

Northeast

\section{Payer type, n (\%)}

Medicare

$\mathrm{PPO} /$ commercial

$\mathrm{HMO} /$ managed care

Medicaid

Other

Unknown
$168(22.1)$

$53(7.0)$

$278(36.8)$

468 (61.9)

$166(22.0)$

77 (10.2)

$45(6.0)$

$296(39.2)$

234 (30.9)

87 (11.5)

$58(7.7)$

$60(7.9)$

$21(2.8)$

$2 \mathrm{~L}=$ second line; $3 \mathrm{~L}=$ third line; $\mathrm{ECOG}=$ Eastern Cooperative Oncology Group; $\mathrm{HMO}=$ health maintenance organization; $\mathrm{PPO}$ = preferred provider organization; $\mathrm{US}=$ United States; $\mathrm{SD}=$ standard deviation.

Table 2. Third line chemotherapy-utilization by backbone categories and costs.

\begin{tabular}{ccc}
\hline Backbone categories & $\mathbf{N}=\mathbf{5 7 7} \mathbf{n}(\%)^{\mathbf{1}}$ & $\begin{array}{c}\text { Unadjusted average total cost } \\
\text { per patient (US dollars) }\end{array}$ \\
\hline Irinotecan + EGFR & $159(27.56)$ & $13,869.89$ \\
Anti-EGFR-monotherapy & $121(20.97)$ & $13,501.02$ \\
Irinotecan-based (without EGFR) & $82(14.21)$ & 9345.34 \\
Capecitabine-based (without EGFR) & $61(10.57)$ & 5419.77 \\
Oxaliplatin-based (without EGFR) & $58(10.05)$ & $16,464.73$ \\
Multi-drug (without EGFR) & $43(7.45)$ & 8989.48 \\
Others & $34(5.89)$ & $10,327.48$ \\
Capecitabine + EGFR & $7(1.21)$ & $10,261.96$ \\
Multi-drug + EGFR & $6(1.04)$ & $20,209.34$ \\
Oxaliplatin + EGFR & $6(1.04)$ & $17,116.68$ \\
\hline
\end{tabular}

EGFR = epidermal growth factor receptor inhibitor; Categories are mutually exclusive per patient. Multidrug defined as two or more of the chemotherapy backbone categories. Irinotecan and Oxaliplatin-based regimens include bevacizumab. Costs per 2012 Medicare allowable. Total outpatient costs from all procedures from initiation of $3^{\text {rd }}$ line therapy until earliest endpoint of last chemotherapy administration plus at least 30 days, or progression, last contact or death. Cost information unavailable for $28 \%$ of patients; oral therapies not included.

those with known KRAS results, $74 \%$ were wild type and $26 \%$ were mutant.

\subsection{Clinical Outcomes}

Time to treatment discontinuation (TTD) and overall survival (OS) was evaluated for the third line treatment population according to backbone treatment categories. The 
Kaplan-Meier TTD was not significantly different between groups $(p=0.8724)$. Figure 1 shows the Kaplan-Meier OS curves. No significant difference was shown between the treatment groups $(\mathrm{p}=0.4673$ ). The OS by patient demographic and clinical characteristics at third-line was also not significant between groups except for BMI (normal $=8.8$ months, obese $=14.4$ months, overweight $=11.5$ months; $\mathrm{p}<0.0001$ ), ECOG performance status (ECOG PS $0=12.6$ months, ECOG PS $1=12.3$ months, ECOG PS $\geq 2=$ 6.4 months; $\mathrm{p}<0.0001$ ), and KRAS testing results (mutations $=12.2$ months, wild-type $=$ 13.3 months, unknown $=9.0$ months; $\mathrm{p}=0.0049$ ). When evaluated by whether patients were adherent or non-adherent to NCCN guidelines in third-line therapy, outcome results were consistent regardless of adherence status.

\subsection{Economic Assessment}

Using 2012 Medicare allowable costs, the unadjusted average total cost of outpatient treatment services provided from start of third-line therapy to endpoint (progression, last contact, or death) ranged from means of $\$ 5419$ to $\$ 20,209$ depending on backbone treatment category (capecitabine-based and multi-drug + EGFR, respectively, Table 2). The unadjusted total cost per patient when evaluated by adherence to NCCN Guidelines was significantly different (median cost $\$ 11,998$ and $\$ 8790$ for adherence vs nonadherence respectively; $\mathrm{p}=0.0007$ ).

The multivariable determinants of cost in third-line therapy showed that there were no significant predictors of total cost in this model. However, in univariate analysis of

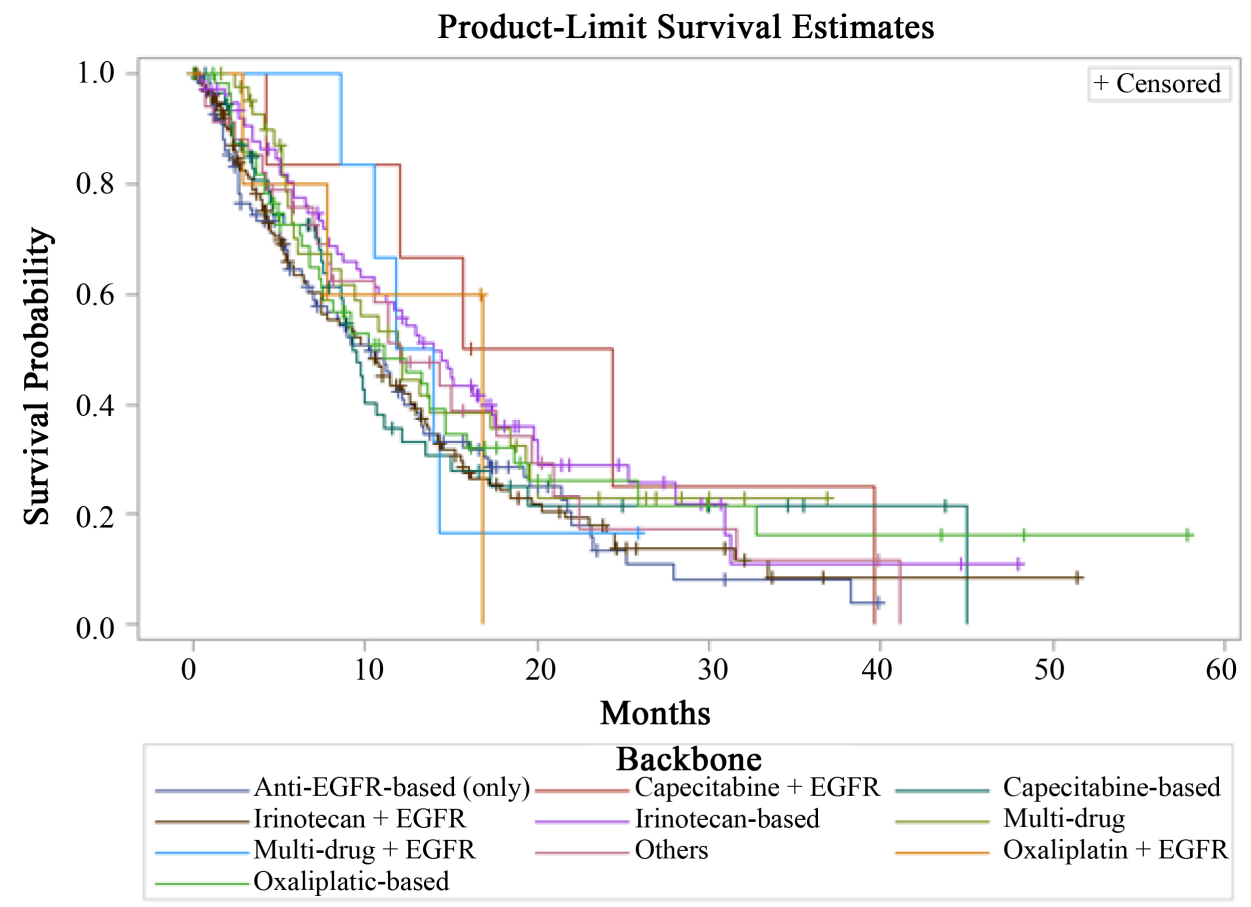

Log-Rank test P-value $=0.4673$

Figure 1. Overall survival by chemotherapy backbone and third-line therapy. EGFR = epidermal growth factor receptor inhibitor. 
total costs, female gender and KRAS mutations were significant predictors of reduced total cost, while NCCN adherence was not a predictor.

\section{Discussion}

This study was designed to assess treatment patterns and outcomes in patients with metastatic colorectal cancer, with a focus on patients who received treatment with third-line therapy. The demographic and patient characteristics of mCRC patients treated in the community setting appear to be similar to that reported in other studies [7] [8]. The most commonly utilized regimen in the first, second, and third line settings during this study period was FOLFOX + bevacizumab, FOLFIRI + bevacizumab, and irinotecan + cetuximab respectively, demonstrating high utilization of monoclonal antibody targeted therapies in each line. In another recently reported large EHR study of chemotherapy usage patterns in a US cohort of patients with mCRC, including academic, private, and community based oncology practices, fluoropyrimidine/oxaliplatin combination was the most commonly used first-line chemotherapy regimen, representing $71 \%$ of first-line therapy by 2007 , and first-line bevacizumab use averaged $51 \%$. Of those who received first-line bevacizumab, $34 \%$ continued to receive bevacizumab in the second-line [7].

The majority of patients received on-pathway and guideline recommended regimens during the first-line setting, although adherence dropped with subsequent lines. Other studies that have retrospectively evaluated adherence to guidelines or pathways for colorectal cancer have reported adherence rates to the initial primary therapy only, with reported adherence rates of $83 \%$ [5] [9]. Our study results of $88 \%$ and $84 \%$ adherence in the first line setting to NCCN guidelines and the US Oncology pathways respectively are consistent with that previously reported in the literature. Compliance thresholds in other cancer pathway programs for colon cancer have also been reported at $80 \%$ [10]. This study however provides additional information regarding adherence rates with later lines of therapy. The lower adherence with subsequent lines may potentially reflect differences in sequencing preferences and increased variability of treatment with later lines. This study encompassed a 5-year time frame, however adherence was retrospectively assessed based on one version of the guidelines, and was not assessed real-time when the treatments were selected. Of note, clinical trial patients were also excluded from this analysis, these patients would be considered on-pathway for US Oncology. The KRAS status was known for $65 \%$ of patients, of which $74 \%$ were wild-type. This is slightly higher than the $60 \%$ of patients with wild-type reported in the literature [4]. This reflects what was documented as structured text in the EHR. If the status was dictated or documented in the progress note as free text, it was not captured. Therefore the documentation rate may be underreported; however, in those patients with a known documented KRAS status, documentation of status occurred most frequently prior to the third-line setting, reflecting its use as a determinant of treatment selection in this line. In another study of KRAS testing in $\mathrm{mCRC}, 50 \%$ of eligible patients were not tested for KRAS [11]. Of note, midway during the study period in 2009, the NCCN 
treatment guidelines were updated to recommend KRAS testing at time of diagnosis for patients with mCRC. This may also account for the lower percentage of patients with known KRAS status during this study. Global labeling and guidelines have since changed to address RAS testing (as opposed to just KRAS testing) for EGFR inhibitors.

Overall survival in the third-line setting did not differ when assessed by the different backbone treatment categories, although this should be considered exploratory as patients were not matched for disease severity. BMI and KRAS were significant predictors of survival in this setting.

The strengths of our study include the large, multisite sample population of advanced mCRC patients from the community treated with advanced lines of therapy. This study was also able to utilize both combined clinical information available from the EHR and cost information from claims.

Limitations of our study included the retrospective nature of the data collection and the potential for documentation bias if there were errors or omissions in the clinical record. We also excluded patients who were participating in clinical trials, as the structure of the trials and their experimental treatments may have impacted the overall findings from our non-experimental population. As treatment for mCRC generally continues until progression or toxicity, progression was defined if a patient was identified as having a subsequent line of therapy. However, a limitation of this approach is that patients may stop a regimen due to toxicity. Assessments of healthcare utilization and costs were from an outpatient perspective, therefore inpatient utilization and costs were not included. Additionally, oral drugs (if not administered by the practice) were not captured. These results may not be generalized to the entire US population as not all community practices utilize iKM, and the majority of practices included were from the southern US.

Costs in the third-line did appear to differ when assessed by adherence to NCCN guidelines. This could be due to incorporation of newly-approved drugs into later lines and refractory settings, and incorporation of these newer, more expensive drugs into the guidelines. Costs reflect Medicare allowable 2012 charges for procedures that occurred during the third-line from the practice outpatient setting. Costs of procedures that occurred outside of the clinic were not captured, as well as costs for oral drugs. In this analysis, $28 \%$ of the patients were not mapped from iKM to the claims data therefore are not included in the cost analyses; however, average and median total costs reported reflect those that were captured from the majority of patients.

This was a retrospective, observational study providing insight into the real world patterns of care and economic assessment of mCRC patients treated in the community. The treatment patterns and clinical outcomes reflect the specific study time frame, from 2007 through 2012. Extrapolation to other treatment periods will be limited by changes in therapy during that time, and with the emergence of new scientific data.

\section{Conclusion}

As new agents continue to emerge, and agents approved for later lines of therapy get 
integrated earlier into the trajectory of care, it is important to understand previous sequencing choices and outcomes to help inform future selection options. As the treatment options for later lines of therapy are influenced by a host of factors such as prior therapy, comorbidities, and insurance coverage, the use of on-guideline regimens are encouraged to support the use of evidence-based medicine.

\section{References}

[1] Siegel, R.L., Miller, K.D. and Jemal, A. (2015) Cancer Statistics. CA: A Cancer Journal for Clinicians, 65, 5-29. http://dx.doi.org/10.3322/caac.21254

[2] (2015) National Cancer Institute Surveillance, Epidemiology and End Results Program. http://seer.cancer.gov

[3] (2013) National Comprehensive Cancer Network Clinical Practice Guidelines for Colon Cancer v3. http://nccn.org

[4] Fakih, M.G. (2015) Metastatic Colorectal Cancer: Current State and Future Directions. Journal of Clinical Oncology, 33, 1809-1824. http://dx.doi.org/10.1200/JCO.2014.59.7633

[5] Hoverman, J.R., Cartwright, T.H., Patt, D.A., et al. (2011) Pathways, Outcomes, and Costs in Colon Cancer: Retrospective Evaluations in 2 Distinct Databases. American Journal of Managed Care, 17, SP45-SP52. http://dx.doi.org/10.1200/jop.2011.000318

[6] Gill, S., Dowden, S., Colwell, B., et al. (2014) Navigating Later Lines of Treatment for Advanced Colorectal Cancer-Optimizing Targeted Biological Therapies to Improve Outcomes. Cancer Treatment Reviews, 40, 1171-1181. http://dx.doi.org/10.1016/j.ctrv.2014.10.002

[7] Abrams, T.A., Meyer, G., Schrag, D., et al. (2014) Chemotherapy Usage Patterns in a USWide Cohort of Patients with Metastatic Colorectal Cancer. Journal of the National Cancer Institute, 106, djt371. http://dx.doi.org/10.1093/jnci/djt371

[8] Landsman-Blumberg, P.B., Carter, G.C., Johnson, B.H., et al. (2014) Metastatic Colorectal Cancer Treatment Patterns According to Kirsten Rat Sarcoma Viral Oncogene Homolog Genotype in U.S. Community-Based Oncology Practices. Clinical Colorectal Cancer, 13, 178-184. http://dx.doi.org/10.1016/j.clcc.2014.05.001

[9] Hines, R.B., Barrett, A., Twumasi-Ankrah, P., et al. (2015) Predictors of Guideline Treatment Nonadherence and the Impact on Survival in Patients with Colorectal Cancer. Journal of the National Comprehensive Cancer Network, 13, 51-60.

[10] Feinberg, B.A., Lang, J., Grzegorczyk, J., et al. (2012) Implementation of Cancer Clinical Care Pathways: A Successful Model of Collaboration between Payers and Providers. Journal of Oncology Practice, 8, e38s-e43s. http://dx.doi.org/10.1200/JOP.2012.000564

[11] Carter, G.C., Landsman-Blumberg, P.B., Johnson, B.H., et al. (2015) KRAS Testing of Patients with Metastatic Colorectal Cancer in a Community-Based Oncology Setting: A Retrospective Database Analysis. Journal of Experimental \& Clinical Cancer Research, 34, 29. http://dx.doi.org/10.1186/s13046-015-0146-5 
Submit or recommend next manuscript to SCIRP and we will provide best service for you:

Accepting pre-submission inquiries through Email, Facebook, LinkedIn, Twitter, etc. A wide selection of journals (inclusive of 9 subjects, more than 200 journals)

Providing 24-hour high-quality service

User-friendly online submission system

Fair and swift peer-review system

Efficient typesetting and proofreading procedure

Display of the result of downloads and visits, as well as the number of cited articles

Maximum dissemination of your research work

Submit your manuscript at: http://papersubmission.scirp.org/ 